\title{
Política y crisis en América Latina: Reacción e impacto frente a la Covid-19
}

\author{
Salvador Martí i Puig y Manuel Alcántara Sáez (Editores)
}

Marcial Pons. 2020. 404 páginas.

\section{Julieta Bertolini}

https://doi.org/10.46468/rsaap.15.1.R5

Política y crisis en América Latina: Reacción e impacto frente a la Covid-19 constituye un destacado análisis inicial de las políticas públicas en respuesta a esta crisis sanitaria en América Latina. Su objetivo primordial es comprender qué factores pueden explicar la realidad de la extensión de la pandemia en cada país. El libro presenta, así, un arduo ejercicio colectivo de investigación y política comparada en 19 países de la región, centrándose en cuatro grandes temas: la comunicación, el liderazgo, las políticas de salud y economía, y la gestión respecto a los derechos, el territorio y la seguridad. Cabe destacar que el trabajo abarca temporalmente el primer semestre de la pandemia, entre febrero (cuando aparecieron los primeros contagios en la región) y julio de 2020.

La introducción por parte de los editores presenta la aparición inesperada de la pandemia como una (potencial) nueva coyuntura crítica-siguiendo la tradicional teoría de path dependence de Collier y Collier (1991)- para la región, entendiendo que muchas de las dinámicas políticas generadas por esta crisis pueden transformar conductas e incluso acuerdos institucionales que perdurarán. Por otra parte, y de manera acertada, los editores resaltan la complejidad en la producción de datos relacionados con la Covid-19, particularmente en una región donde la capacidad estatal de publicar estadísticas confiables es pobre. Esto es relevante en tanto el éxito de la respuesta sanitaria se ha evaluado principalmente a través de la evolución de los números diarios de contagiados y fallecidos.

Le siguen a la introducción 19 capítulos -uno por cada caso nacional- escritos por académicos locales o especialistas en el país asignado, lo que ha asegurado un altísimo nivel de conocimiento y experiencia en el análisis. Los estudios de casos se basan en los cuatro temas centrales mencionados anteriormente, facilitando la comparación, pero también realzando las particularidades de cada caso con información y apreciaciones más detalladas.

Lara Goyburu se centra en el fenómeno de "la grieta" en Argentina, resaltando el fortalecimiento de la imagen presidencial a sólo tres meses de su comienzo. Franz Flores Castro estudia cómo Bolivia enfrentó la crisis sanitaria durante el gobierno transitorio de Jeanine Áñez. Michelle Fernandez y Humberto Dantas analizan el entonces ya complejo escenario en Brasil, a causa de la ausencia de liderazgo de Jair Bolsonaro. Gonzalo Delamaza describe la errática gestión inicial en Chile, que resultó en la renuncia del ministro de salud en junio y en una nueva estrategia desde entonces. Porfirio Cardona-Restrepo, Luis Guillermo Patiño Aristizábal y Luz Mery Rojas Cárdenas analizan la gestión colombiana, en tanto María José Cascante y Eugenia Aguirre Raftacco buscan comprender los factores detrás de los buenos resultados en materia sanitaria y el 
consistente apoyo al gobierno en Costa Rica. Arturo López-Levy y Daniel Rodríguez Suárez evalúan el efecto de la Covid-19 en Cuba en torno a tres dimensiones: el reto sanitario, el desafío político y la recuperación económica. Angélica Abad Cisneros y María José Calderón analizan la crisis en Ecuador, uno de los países más golpeados ante la incapacidad gubernamental. Alberto Martín Álvarez resalta el autoritarismo y los rasgos populistas que marcan la gestión de Nayib Bukele en El Salvador, a la vez que Juana M. Guerrero Guernica reconstruye la gestión frente a la crisis sanitaria en una Guatemala colapsada económicamente. Carlos Barrachina Lisón muestra cómo la emergencia de la Covid-19 afecta a un país pobre de débiles instituciones, altos niveles de corrupción y profunda alarma social como Honduras. Alberto Aziz Nassif explica cómo la pandemia agudizó la polarización y creó un nuevo estilo de gobierno en México, mientras Mateo Jarquín estudia el impacto de la crisis en Nicaragua, en medio de tensiones sociales, deterioro económico y una violenta consolidación del autoritarismo en manos de los OrtegaMurillo. Harry Brown Araúz revisa los efectos de la desigualdad en la gestión de la crisis sanitaria en Panamá. Sara Mabel Villaba hace hincapié en la verdadera emergencia paraguaya: la corrupción, el autoritarismo y la violencia policial. Adriana Urrutia y Núria Sala i Vila dan cuenta de los profundos problemas estructurales que sufre Perú, a la vez que pronostican los efectos de la crisis en la carrera presidencial de 2021. En tono similar, Omar Pérez-Rubiera también resalta la coyuntura electoral en República Dominicana, mientras Pablo Brugnoni, Adolfo Garcé y Rafael Porzecanski presentan el gran caso de éxito (hasta entonces) en la gestión sani- taria de la región: Uruguay. Finalmente, Margarita López Maya describe la evolución de la gestión de Nicolás Maduro en Venezuela, marcada por una grave crisis humanitaria y una absoluta crisis política.

La conclusión presenta una clasificación sumamente útil (que funcionaría mejor en la introducción) de los potenciales factores para evaluar la efectividad de la lucha contra de la pandemia en cada país: contextuales (capacidad estatal general y en atención primaria de salud, informalidad, infraestructura de vivienda y grado de madurez cívica), de condición (grado de aislamiento externo y tamaño poblacional) y coyunturales (tipo y calidad de liderazgo y estilo de comunicación, tipo de políticas de respuesta a la crisis, capacidad de clausura de las fronteras nacionales y de restricción a la circulación interna, y disposición de recursos sanitarios).

Luego, expone un ejercicio comparativo de los 19 casos centrándose en dos de los cuatro temas destacados. El análisis de la comunicación se resume en cinco variables: si estuvo centralizada en el presidente, si hubo presencia de actores relevantes y, en tal caso, cuáles (por ejemplo, el Ministerio de Salud o el Jefe de Gobierno), si el discurso oficial adoptó tintes bélicos y/o religiosos, si la estrategia se mantuvo en el período, y cuáles fueron los medios preferentes (TV, radio, redes). En cuanto al liderazgo institucional, comparan el activismo del presidente de la república (y su imagen positiva) y el de los tres poderes gubernamentales. Las políticas de salud y economía y la gestión del territorio y la seguridad ocupan un espacio menor hacia el final, comparando principalmente las medidas de restricción a la circulación interna y externa. El capítulo suma además como factor el papel de los actores 
políticos y sociales -partidos de la oposición, medios de comunicación privados, sociedad civil organizada e iniciativas comunitarias- en la crisis.

El libro cierra con una interesante y práctica revisión bibliográfica a cargo de Anaïs Varo Barranco en torno a los debates principales de la pandemia en la región: el impacto de la política, la desigualdad y los derechos humanos (enfocándose en la resiliencia de los sistemas de salud, la desigualdad en las políticas públicas y las violencias durante la crisis: militarización de las calles, fronteras y género), la importancia de los liderazgos y los actores políticos, y los impactos económicos y comerciales de la Covid-19.

Mucho ha cambiado desde que el libro fue redactado; las conclusiones que se presentan en el capítulo final quedan, en parte, "obsoletas". Dos países que los editores resaltan por su efectividad, Uruguay y Paraguay, se ven hoy en una situación sanitaria crítica. La región se ve actualmente inserta en la carrera mundial por la vacunación de toda la población (o, al menos, de la población de riesgo), en un contexto de profunda desigualdad mundial en la producción y el reparto de dosis. Así, América Latina está entrando lentamente en una segunda (o tercera) ola, con un revival de las medidas que este libro analiza.

Es de destacar, sin embargo, la precisión analítica y el volumen de información recabada por los escritores en tan corto tiempo, lo que hace de este libro un trabajo admirable. Podrían pensarse varias reediciones posteriores, con el estímulo de ir incorporando en la reconstrucción de las respuestas a la crisis de la Covid-19 los nuevos y vertiginosos acontecimientos que surgen en la región y en el mundo casi día a día. Así, podría confirmarse si esta pandemia ha sido, efectivamente, un momento de coyuntura crítica. Este trabajo debe ser, por lo tanto, celebrado, ya que brinda un excelente primer análisis de las políticas públicas implementadas durante los primeros meses de la pandemia, y será una referencia ineludible para quienes las estudien en la actualidad o en el futuro.

\section{Referencias bibliográficas:}

Berins Collier, R. y D. Collier. (1991). Shaping the political arena: critical junctures, the labor movement and regime dynamics in Latin America. Princeton: Princeton University Press. 